.

\section{A COMMENTARY ON THE INSTRUMENTATION FOR BUILDING ENERGY MONITORING AND CONTROL SYSTEMS (EMCS)}

James Y. Kao

David W. Baker

Building Equipment Division Center for Building Technology National Bureau of Standards U.S. Department of Commerce Washington, DC 20234

September 1980

Sponsored by:

Civil Engineering Laboratory

Naval Construction Battalion Center

Port Hueneme, CA

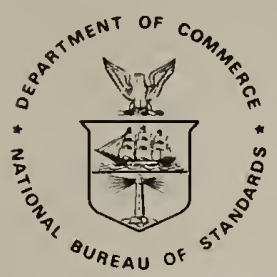

U.S. DEPARTMENT OF COMMERCE, Philip M. Klutznick, Secretary

Luther H. Hodges, Jr., Deputy Secretary

Jordan J. Baruch, Assistant Secretary for Productivity, Technology, and Innovation

NATIONAL BUREAU OF STANDARDS, Ernest Ambler, Director 



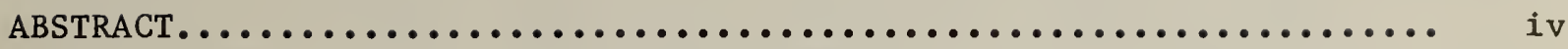

CONVERSION FACTORS TO METRIC (SI) UNITS.................... v

1.0 STEAM FLOW MEASUREMENTS............................. 1

1.1 ORIFICES AND FLOW NOZZLES......................... 1

1.2 VORTEX SHEDDING METER........................... 5

2.0 HOT WATER AND CHILLED WATER FLOW MEASUREMENTS .............. 7

2.1 ORIFICES, FLOW NOZZLES, AND VENTURIS................. 7

2.2 VORTEX SHEDDING METER............................. 7

2.3 TURBINE METER.................................. 7

3.0 TEMPERATURE MEASUREMENTS.............................. 10

3.1 LIQUID-IN-GLASS THERMOMETERS ....................... 10

3.2 RESISTANCE THERMOMETERS........................... 12

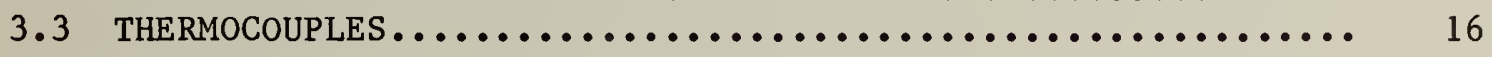

4.0 HUMIDITY MEASUREMENTS................................ 18

4.1 SALT-PHASE TRANSITION TYPE HYGROMETER................. 18

4.2 IMPEDANCE TYPE HYGROMETER............................ 19

4.3 DIMENSIONAL CHANGE HYGROMETER........................ 20

REFERENCES.............................................. 22 



\section{ABSTRACT}

This paper reviews sensors generally used for building energy monitoring and control systems (EMCS). The sensor operating principles, performances, calibration, maintenance, installation precautions, failure modes and their suitability for building EMCS use are discussed. Sensors covered in the paper include orifices, flow nozzles, Venturis, votex shedding meters, and turbine meters for flow measurements, liquid-in-glass thermometers, resistance thermometers, and thermocouples for temperature measurements, and salt-phase transition hygrometers, impedence hygrometers, and dimensional change hygrometers for humidity measurements.

Key words: building energy monitoring; instrumentation; sensors. 
CONVERSION FACTORS TO METRIC (SI) UNITS

\begin{tabular}{lcccc}
$\begin{array}{l}\text { Physical } \\
\text { Quantity }\end{array}$ & Symbol & $\begin{array}{c}\text { To Convert } \\
\text { From }\end{array}$ & To & Multiply By \\
\hline Length & & $\mathrm{ft}$ & $\mathrm{m}$ & $3.048 \times 10^{-1}$ \\
Temperature & $t$ & ${ }^{\circ} \mathrm{F}$ & ${ }^{\circ} \mathrm{C}$ & ${ }_{\mathrm{t}_{\mathrm{c}}}=\left(\mathrm{t}_{\mathrm{f}}-32\right) / 1.8$ \\
Density & $\rho$ & $1 \mathrm{~b} / \mathrm{ft}^{3}$ & $\mathrm{~kg} / \mathrm{m}^{3}$ & $1.602 \times 10^{+1}$ \\
Kinematic Viscosity & $\nu$ & $\mathrm{ft}^{2} / \mathrm{s}$ & $\mathrm{m}^{2} / \mathrm{s}$ & $9.290 \times 10^{-2}$ \\
Mass Flowrate & $\mathrm{M}$ & $1 \mathrm{~b} / \mathrm{hr}$ & $\mathrm{kg} / \mathrm{hr}$ & 0.4536 \\
Pressure & & $\mathrm{Psi}$ & $\mathrm{Pa}$ & $6.895 \times 10^{3}$ \\
Velocity & & $\mathrm{ft} / \mathrm{min}$ & $\mathrm{m} / \mathrm{s}$ & $5.080 \times 10^{-3}$ \\
Volumetric Flowrate & & $\mathrm{GPM}$ & $\mathrm{m}^{3} / \mathrm{s}$ & $6.309 \times 10^{-5}$
\end{tabular}




\subsection{ORIFICES AND FLOW NOZZLES}

Operating Principles. These devices measure flowrate, converting kinetic energy to a pressure differential. The equation may be written:

$$
M=358.93 \mathrm{CYd}^{2} \mathrm{~F}_{\mathrm{a}} \sqrt{\rho \mathrm{h}_{\mathrm{w}}} / \sqrt{1-\beta^{4}} \text {, where }
$$

M Mass flowrate, $1 \mathrm{~b} / \mathrm{hr}$

C Coefficient of discharge for the orifice nozzle

$\mathrm{Y}$ expansion factor (fluid expansion)

d diameter of orifice, inches

$\mathrm{F}_{\mathrm{a}}$ area factor for thermal expansion of orifice

$\rho$ density of steam entering meter, $1 \mathrm{~b} / \mathrm{ft}^{3}$

$h_{w}$ differential pressure, inches of water at $68^{\circ} \mathrm{F}$

$B d / D$, where $D$ is the inside diameter of the pipe

Measured differential pressure $h_{w}$ (full scale value) typically varies from a few inches of water to 10 or 20 psi. Low values of $h_{w}$ result in lower (pumping energy) losses and a smaller variation in steam expansion factor $Y$ (for a given steam pressure level), but low values are more difficult to measure accurately. Pressure losses are nominally lower for nozzles as manifested through values of $C$, ranging from about 0.60 to 0.65 for orifices and from 0.93 to 1.0 for nozzles. Values of $Y$ and $C$ are available for square edge concentric bore orifices and flow nozzles $[1,2]$ *.

For metering wet steam in horizontal lines, eccentric or segmental orifice plates are often used, although metering in vertical lines using concentric orifice plates is preferred [2]. In any case, the accurary will be less when metering wet steam.

Performance. Accuracy or uncertainty is considered to be comprised of two components, that due to the systematic error and that due to the precision or random error. For direct calibration of the overall meter system which includes the differential pressure transmitter system, the upstream and downstream piping and suitable flow straightener, best accurary is estimated to fall within the approximate range 0.2 to 0.5 percent of rate when flowing steam during the calibration at the same temperature and pressure conditions which will exist when the meter is used on-line. For meters not receiving a direct calibration, the estimated accurary value will range from perhaps 1 to 2 percent and upward. This value is strongly dependent on the differential pressure transmitter system characteristics. Also, the tolerance of $\mathrm{C}$ and $\mathrm{Y}$ influence the accuracy, as well as how the variations of $\mathrm{C}$ with Reynolds number and $Y$ with $h_{W}$ are treated. Most industrial type differential pressure transmitter systems are usually rated on a full-scale basis, and may be quite sensitive to flow range such as $3: 1$ ( $9: 1$ range in $h_{W}$ ), or is subjected to just normal ambient temperature changes, the metering accuracy

* Numerals in brackets refer to references cited at the end of the report. 
can deteriorate significantly unless special precautions are taken. Such precautions would include establishing (and malntaining) the transmitter performance through calibration so that non-linear effects could be taken into account, and possibly operating the transmitter in a constant temperature environment. Whether such precautions are warranted depends on individual application requirements. However, for systems where steam flo rates and/or operating conditions vary significantly, serious consideratic. should be given to incorporation of data and calibration results into tho software of the computer based energy monitoring and control system (EMCC) to take into account variations in $C, Y, F_{a}$, and $\rho$ with measured flow conditions.

The response time between $\Delta \mathrm{P}$ changes and systems output, neglecting data read and processing time, depends mainly on transmitter characteristics. From our experience in a current bullding EMCS program at the National Bureau of Standards (NBS), the transmitter force balance systems typicali: had response times of about 1 second or less.

The performance parameters "repeatability," hysteresis and deadband are $\hat{\perp} 1$ considered part of the precision error of the transmitter, since in use the direction of pressure change will be random. Industrial type trans" mitters are typically rated at $0.1 \%$ to $0.2 \%$ of span for these parameters.

Calibration and Maintenance. The orifice plate and nozzle themselves require no maintenance. However, periodic inspection of the main line for dirt and the orifice plate sharp edge for pitting and other damage is advisable. Also, the pressure sensing lines should be inspected and checked for leakage, and the pressure taps for dirt and plugging. The pressure transmitter system should be periodically vented and checked for alr and vapor, and then calibrated as installed on line, if possible. This should include at least a zero $\Delta \mathrm{P}$ check and check of several $\Delta \mathrm{P}$ inputs between zero and full scale. Performing these calibration checks at line operating pressure level is preferred. If. this is not possible, then the zero $\Delta \mathrm{P}$ checks should include one at a line pressure and one at the calibration pressure level. The transmitter should perform according to the manufacturer's specification and a calibration history or $\log$ should be maintained for each transducer, using perhaps a control chart or suitable approach. As a favorable history of a transmitter performance is accumulated, the time interval between calibrations can be extended. Before the government "buys the system," the pressure transmitter equipment should be checked for normal and stable operation and a calibration performed. Orifice plates and nozzles should be inspected and dimensions (orifices and nozzle throat bore) checked closely.

Installation Precautions. To minimize the possibility of large errors in the flow measurement due to transverse or swirling flow components, the primary element (orifice plate or nozzle) should be installed in a straight pipe run with a suitable flow straightening vane assembly upstream of this element. As minimum requirements, see the recommendations of [1], Chapter 
II-II. The importance of flow pattern control (flow straightening in particular) cannot be over-emphasized for accurate metering.

The installation of the pressure taps and pressure sensing line system is especially important in metering steam because of the large density difference between the flowing vapor and the condensed liquid in the sensing lines. The interface between steam and vapor and liquid in each leg must be at the same height and the effective density of the liquid should be the same in each line. Good practice dictates keeping these lines short and close together, with a slope of at least 1 inch per foot. Reference 1 includes installation details for both horizontal and non-horizontal runs including use of reservoirs where necessary. In addition, to eliminate trapped air and vapors, it is recommended that a vent valve or plug be installed at the highest point in each line or leg, along with the drain valve or plug at the lowest point in each. To ensure a positive check on bypass leakage, a " $T$ " valve network using three valves between the high and low pressure legs is recommended. The "top" of the $T$ is connected between the legs and includes two sealing valves, one in each side of the "top." A vent valve normally open to atmosphere is included in the "leg" of the $\mathrm{T}$. In this way, any bypass leakage will be indicated as leakage through the vent valve. Figure 1 shows a general arrangement of the piping valves and fittings.

Failure Modes. No complete or catastrophic failures or differential pressure transmitter units have occurred in the present NBS program. However, apparent drift of the zero balance or slope characteristics of the transmitter does occur. If, after checking the pressure sensing lines, venting, draining and allowing sufficient time for the lines to fill with condensate and reach steady temperature, the apparent trouble persists and is repeatable, the trouble is assumed in the transmitter and its performance is investigated.

Building EMCS Suitability. Orifices and nozzles have long been used for metering fluids in the process industries and building systems. The $\Delta \mathrm{P}$ sensing instrumentation have included use of (direct head sensing) glass tube manometer, fluid mechanical pressure measuring transducer/transmitter readout systems, and electronic pressure measuring systems evolving from the vacuum tube era to the more accurate, low maintenance solid state systems in use today. Thus, a great deal of experience and knowledge have been accumulated on both the primary metering elements and the $\Delta \mathrm{P}$ sensing transmitter systems. Today, there are sufficient sources of complete metering systems, or primary element and pressure transmitter systems separately, which are available at reasonable cost and capable of good accuracy. Further, this type of meter has no moving parts in the primary element, and can perhaps tolerate "dirtier" fluids compared with those having moving parts. All of these factors contribute to making such systems suitable for EMCS applications.

However, for a given meter configuration, the fluid properties, flow conditions and line piping geometry all influence the pressure differential along with the fluid flowrate itself. This results in a complex measurement situation comprised of two "problems": that concerning hardware, i.e. the installation, calibration and maintenance of systems in accordance 


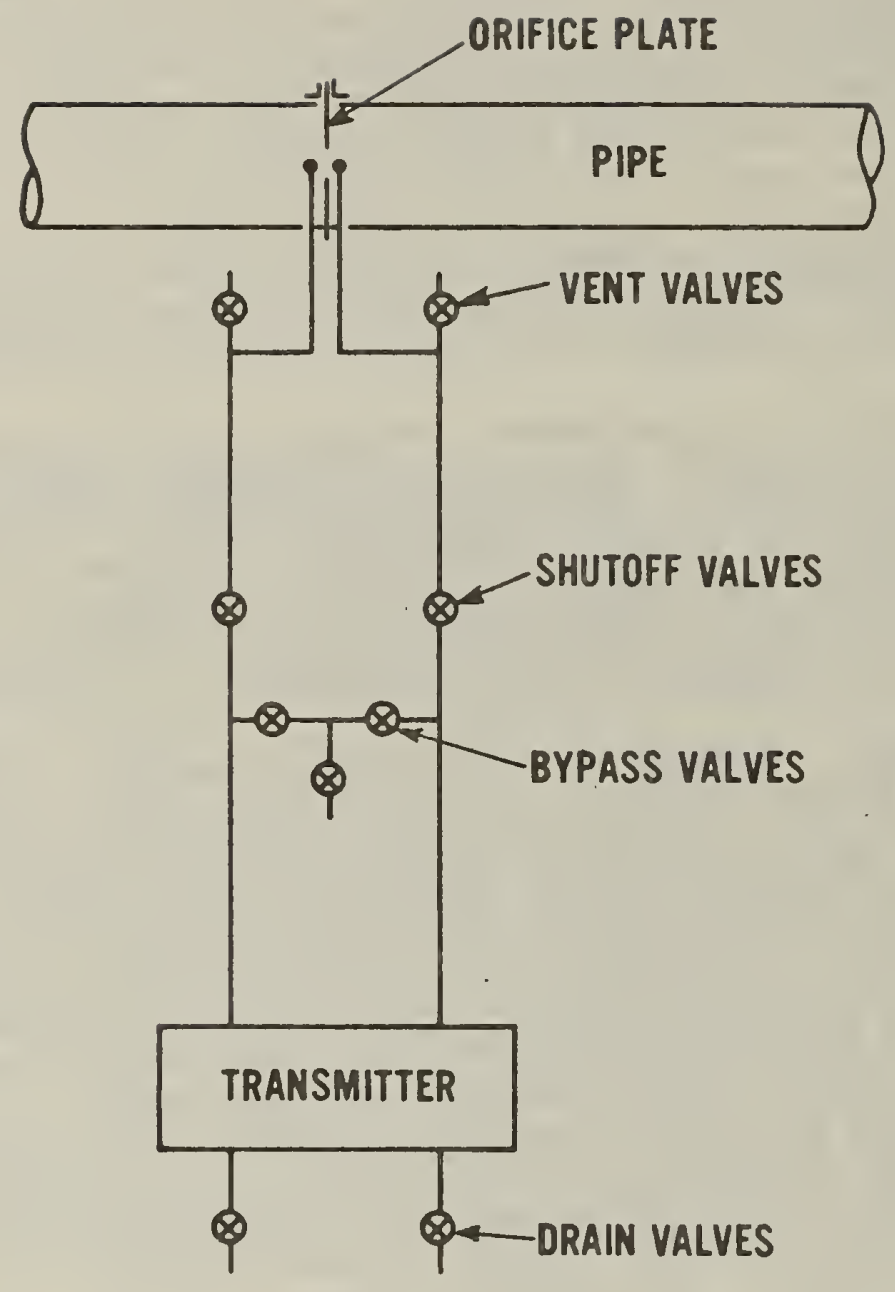

Figure 1. Orifice plate sensing line valve arrangement 
with good metering and instrumentation measurement principles, and that concerning, software, i.e. the processing, calculation, storage and output of (large amounts of) parameter data and resulting flow data. In this sense, this type meter is unsuitable as a convenient means of measuring flow. Also, the accuracy of the indicated readings during partial load conditions is degraded, since the accuracy characteristics of the transmitter systems are often rated on a full scale or span basis.

The use of digital computer-based data processing can solve the software problem outlined above. Also, through the computer, the accuracy degradation situation can be improved significantly provided sufficient calibration data is provided periodically and a performance "curve" is established for each transmitter. The hardware problem as outlined must, as in the past, be solved through good installation and maintenance practices, and where possible, through use of direct calibration techniques. The basic hardware problems here are solved independently of the computer. Wherever feasible, new construction in particular should include provisions for on-line, direct calibration of working meters.

In summary, orifices and nozzles are recommended as a best choice for metering steam in the gaseous state whenever direct calibration services for the meter system including the primary element are unavailable. If calibration services are available for flowing steam at the required temperature and pressure, the vortex shedder type discussed below is recommended as a very promising candidate. This meter lack moving parts and its output is digital in nature. If the application allows, metering of building system condensate should be considered since metering of liquids presents fewer problems than metering of gases.

\subsection{VORTEX SHEDDING METER}

Operating Principles. This meter, on the industrial scene about 10 years, operates on the principles that the frequency of vortex shedding for fluid flow around a submersed object is proportional to the fluid stream velocity. Flowrate is measured by detecting this frequency. A big advantage for a building EMCS application is that accurate measurement of the probe output is a much simpler measurement task than accurate measurement of, for example, the $\Delta P\left(h_{W}\right)$ from a differential pressure type meter. At the present state of the art, meter configurations are available for both gases and liquids in pipe sizes one inch and up, and at temperatures up to about 400 or $500^{\circ} \mathrm{F}$. Meter output is usually expressed in dimensionless terms in functional form:

$$
\mathrm{fD} / \mathrm{V}=\phi(\mathrm{DV} \rho / \mu) \text {, where }
$$

$\begin{array}{ll}\mathrm{fD} / \mathrm{v} & \text { Strouhal number } \\ \mathrm{DV} \rho / \mu & \text { pipe Reynolds number } \\ \mathrm{V} & \text { steam velocity } \\ \mathrm{D} & \text { characteristics linear dimension of the meter } \\ \mathrm{f} & \text { frequency of vortex shedding } \\ \rho & \text { fluid density } \\ \mu & \text { fluid absolute viscosity. }\end{array}$


The function $\phi$ is determined by calibration for each geometrical shape of meter in the same sense that the coefficient of discharge $C$ for an orifice is determined experimentally for each geometry (such as concentric orifice, pipe diameter, orifice diameter, pressure tap configuration). The meter is a volumetric type as evidenced from the Strouhal number $f D / V$, where $V$ is proportional to $Q$, the volumetric flowrate.

Performance. At Reynolds number greater than 15,000, the calibration factsr $R$, such as pulses/gallon or pulses/ft $\mathrm{t}^{3}$ is essentially constant within $0.5 \mathrm{t}$. $2 \%$ for flowrate ranges varying from about $10: 1$ to $100: 1$, depending upon manufacturers' specifications. Pressure losses at rated flow can vary frcm a few inches of water to a few psi depending upon the fluid. Pulse output frequencies are "low." For example, a particular 2-inch meter in water flowing at a rate of 100 GPM produced an output pulse frequency of about $50 \mathrm{~Hz}$. When higher resolution is desired, period measurement techniques (time to count say 10 pulses) can be used. In comparison, when meterial over flowrate ranges greater than $2: 1$ or $3: 1$, orifice meters coupled with full-scale rated $\Delta P$ transducers systems are at a decided disadvantage.

The response time is limited mainly by the electronics circuitry processil the output pulses, which can be as large as several seconds if the signal is converted to a DC voltage. In pulse counting, the count time interval in a sense, represents the response time. With adequate electronics circuitry processing the pulses, the instrument "repeatability" is primarily dependent on the steadiness of the flow stream velocity being measured.

Maintenance and Calibration. Since this meter element has no moving parts, its calibration factor should not change appreciably, and it should require no maintenance except as necessary to keep it clean. Without operating experience with this type of meter, we can only comment that meter performance may suffer or degrade when internal passages leading to thermistor elements or shuttle balls (magnetic pick-up type) become clogged or dirty. Maintaining the electronics circuitry is presumed similar to maintaining general laboratory analog, digital counting instruments.

While each manufacturer can no doubt "predict" performance of each of his nodels, there exist no "standard" configurations used industry wide that correspond to those of the orifice meters or flow nozzles. Thus, direct calibration with the fluid at application operating conditions of interest are recommended until such time as calibration factors for standard configuration become well known. This could be considered a disadvantaga particularly when calibration on steam is required.

Installation Precautions. The vortex shedding meter should not be conside:a cure-all for meter installation troubles encountered in building systems Rather, the same general rules as those for orifice meter apply to this met: in terms of straight runs of pipe upstream and downstream and, in some casen, straightening vanes may be needed. Such is needed to minimize effects of transverse velocity components and (abnormal) upstream tubulence on the steady formation of vortices behind the meter obstruction body. 
Failure Modes. In designs using self-heated thermistors, there should be current limiting circuits to guard against thermistor burnout whenever the cooling rate is low (i.e. unit designed for use in water but energized when exposed to air only).

Building EMCS Suitability. This relatively new type meter is considered quite suitable for EMCS applications. The advantages include: (1) output signal is digital in nature, (2) periodic and long term calibration programs and maintenance of instruments such as $\Delta P$ transmitters would be eliminated, (3) once a meter element is calibrated, it should no longer need often repeated calibrations when used with clean fluids, (4) the accuracy is essentially independent of the flowrate.

The meter is recommended as a very promising candidate whenever calibration services are available for flowing steam at the required temperature and pressure. Its output is digital in nature with frequency essentially linear with flowrate. It is classified as a "candidate" since we have yet to use this type in an EMCS program and because of its newness, documentation of performance in the technical literature is still sparse.

\subsection{HOT WATER AND CHILLED WATER FLOW MEASUREMENTS}

\subsection{ORIFICES, FLOW NOZZLES, AND VENTURIS}

Reference is made to the notes on Steam Flow Measurement above. These notes in general apply to metering of hot and chilled water. However, $\mathrm{HW}$ and $\mathrm{CW}$ are essentially incompressible and thus the expansion factor $Y$ has a value of unity. Although there is no phase change between the flowing fluid and the fluid in the pressure sensing legs, the design and installation of the pressure sensing system should receive equal care and attention since accurate sensing of $h_{w}$ is just as essential for accurate metering. In high temperature hot water and high pressure steam systems, insulation of the pressure sensing lines may be necessary. See refernce 1 for installation details for both horizontal and non-horizontal runs.

\subsection{VORTEX SHEDDING METER}

See notes on this meter under Steam Flow Measurement. These notes in general apply to metering of hot and chilled water.

\subsection{TURBINE METER}

Operating Principles. This meter contains a bladed rotor or turbine which rotates at a velocity proportional to volume rate of flow. Most models employ magnetic pick-offs in which the rotor blades vary the reluctance of a magnetic circuit which generates an $\mathrm{AC}$ voltage in the pick-off coil. The frequency is directly proporational to rotor speed. This frequency is sensed as an indication of flow. It can be counted by an electronic counter, or converted to an analog signal using voltage to frequency converter circuits. The calibration factor or meter factor is expressed in electrical pulses generated per unit volume of throughput, e.g. pulses/gallon. The meter factor is 
sensitive to flowrate, fluid density and viscosity, the fluid flow pattern the meter entrance, and the meter orientation. For a meter of a specified shai!, meter performance can be expressed as:

$$
\mathrm{Q} / \mathrm{nD}^{3}=\Phi\left(\mathrm{nD}^{2} / \nu\right)
$$

where: Q volume flowrate

$n$ speed of rotor

$D$ a characteristic linear dimension of the meter

$\nu$ kinematic viscosity of the fluid $=\mu / \rho$

This functional relation $\Phi$, determined by calibration, describes the performil provided retarding forces acting on the rotor (bearing friction, electromagnetic forces) are insignificant and the fluid is incompressible. When considering on particular meter of fixed size and shape, the quantity $D$ is constant and above dimensionless quantities may be reduced to the form

$$
f / Q=\Phi_{1}(f / \nu), \text { pulses/unit volume }
$$

where $\mathrm{f}$ is the pulse frequency.

Performance. The frequency output of a typical meter can be linear within +0.5 percent at its higher rates of flow when operating on low viscosity fluids, such as water. This range of linear operation, i.e. constant meter factor, may extent over a flow range of 10:1 or higher, depending on meter design and size, and liquid viscosity. With water for the range 40 to $450^{\circ} \mathrm{F}$, the kinems viscosity varies from $1.66 \times 10^{-5}$ to $0.16 \times 10^{-5} \mathrm{ft}^{2} / \mathrm{s}$. Thus, the temperatis range needs consideration for each application, and $\mathrm{f} / \mathrm{\nu}$ remains the contro? variable when temperature varies significantly. At the lower rates of flow for each meter, frequency output becomes very nonlinear resulting from the combined effects of retarding forces due to electromagnetic and mechanical loadin. on the turbine as well as changes in the fluid flow regimes (turbulent/laminar) within the meter. There is always some minimum flowrate below which retaraing forces are greater than the fluid forces causing rotation and the rotor cease. to turn.

The pressure drop through the meter depends on design and varies with $\rho Q^{2}$. Typically, this may be 5 to 10 psi at rated flow.

While meter performance in terms of meter factor varies for a particular me with flowrate and viscosity, the repeatability can be typically 0.1 percent better when all factors which are known to influence the perfornance of the meter are controlled. This means operation at a specified value of variab: $\mathrm{f} / \nu$, (or specified flowrate when $\nu$ is constant), retarding forces are negligible or unchanging, and the entrance flow pattern to the meter containc swirling components.

Installation Precautions. The preceding refers to turbine meter operation usin clean fluids. When dirt or particulate matter are entrained in the water, meter performance may be adversely affected due to changes (increases) in the retarding forces, i.e. through "dirty bearings". Therefore, it is important to install 
a suitable filter upstream of the meter and to select a meter with bearings compatible with operation in water.

The dirt problem is one of the common difficulties in using turbine meters, in building systems because these systems may become easily contaminated. The level of contamination tolerable for heating, ventilating and air conditioning (HVAC) equipment may very $1 i k e l y$ be intolerable for turbine meters. Required level of filtration depends on meter size and bearing design. For smaller meters such as one-inch size, a filtration requirement to 50 microns is typical. The manufacturer's recommendations should be followed.

The meter should be installed in a straight length pipe, downstream as far as practical from elbows, valves and the like. For desired accuracy better than one percent, a suitable flow straightener assembly should be installed upstream to eliminate swirling flow components. For best accuracy, the meter and pick-off should both be installed in the same positions as that used during calibration. Minimum pipe installation requirements are given in reference [3].

The pressure level in the meter should be high enough so that cavitation or formation of vapor does not occur. An entrance pressure of 50 psia plus the vapor pressure of the water is usually adequate to overcome pressure losses in the meter and connecting piping, and to prevent cavitation in the meter [4].

Calibration and Maintenance. Unfortunately no single performance curve exists for all turbine meters such as corresponding coefficient of discharge data which exists, for example, for orifice meters. Therefore, each meter must be calibrated to obtain its performance or function given above. For best accuracy, each meter should be calibrated on the liquid to be used during the application. This applies in particular to liquids such as water which has a different lubricity characteristic as compared to liquid hydrocarbons. Instances have been noted when meter performance on water is different from that obtained using liquid hydrocarbons of similar viscosities [4]. Such is apparently due to changes in retarding forces and bearing friction.

Calibration may be performed on a flow meter calibration facility or on-line using suitable transfer reference flowmeters. In either case, for best accuracy, it is important that the flow straightener assembly, the meter and necessary lengths of upstream and downstream piping be calibrated as a unit. Meter calibration should be checked periodically.

Maintenance procedures will include servicing the filter equipment, including changing filters as necessary to avoid excessive flow blockage and/or pressure loss. Also, the meter should be removed from the line periodically and examined for possible solids deposited in the meter.

Failure Modes. Severe overspeeding of the meter should be avoided. Such may occur when venting air from a piping system or by subjecting the meter to very high flowrates above the rated value. High rotor speeds when the meter is dry, in particular, can hasten bearing wear and failure. Subjecting the meter to intense pressure/flow pulsations or mechanical vibration may 
hasten bearing failure and exposure to excessive dirt and solid particales may cause bearing seizure.

Building EMCS Suitability. The turbine meter has advantages of small size, repeatability, and a type of output which is digital in nature. These make the meter quite suitable for EMCS applications. Also, the meter factor is often essentially constant which simplifies the flow calculations. However, the amount of dirt often encountered in building systems and the lubricity characteristic of water raise questions as to its general suitability for this application. Only systems in which contamination can be adequately controlled would appear for this type of meter. Systems which are "norma1? dirty" and in which there is no control over the amount of contamination likely would require a large service effort and equipment expense in maintaining "clean" water for the meter. The lubricity problem is best dealt with be selection of meter bearings suitable for operation on water, and by periodic meter calibration and check. Thus, turbine meters for EMCS applictions are recommended provided the meter is operated on clean water and it can be calibrated on water at the required application temperature and pressure.

\subsection{TEMPERATURE MEASUREMENTS}

\subsection{LIQUID-IN-GLASS THERMOMETERS}

Operating Principles. This very old, we1l-known device depends on the coefficient of expansion of the thermometer liquid being greater than that of the glass bulb and stem. Usefulness in a building EMCS program includes service as reference calibration devices, and for checking temperature measurement equipment on line.

Thermometers are classified into the following types, in accordance with immersion conditions:

Partial Immersion. A reference mark on the stem indicates immersion depth in liquid being measured. The thermometer indicates correctly when immersed to this depth an when the remaining (emergent) portion of the stem is at a specified temperature. When a different immersion depth or a different stem temperature is present, corrections are applied to obtain highest accuracy. See references [5] or [6] for discussion and relations used in making such corrections.

Total Immersion. Indicates correctly when the bulb and entire liquid column is exposed to the temperature being measured, i.e. the top of the thermometer liquid column and the surface of the liquid being measured are at the same level.

Complete Immersion. Indicates correctly when the entire thermometer (including the expansion chamber) is exposed to temperature being measured.

Performance. Typical accuracies for measuring thermometers for EMCS usage vary from values of about $0.1^{\circ} \mathrm{F}$ upward depending upon thermomerer 1 ype, 
temperature range, and scale length. Tables 5 to 10 of reference 5 and Table I of reference 7 are recommended as guideline data for thermometer selection.

The time constant (the time required for an original difference in temperature between thermometer and water bath medium to be reduced to $1 / \mathrm{e}(1 / 2.7$ of itself) for typical solid stem, laboratory type thermometers is 2 to 3 seconds [5]. A settling time (defined as the time required to reach and stay within a stated tolerance band around the final value) of 20 to 30 seconds is sufficient to reach a temperature within 0.01 percent of the initial temperature difference for stirred water immersion. For oil immersion, or still water immersion, settling times should be increased by a factor of two or so. As a consequence, process temperature stability needs consideration for on-line usage. For example, a thermometer having a response time of 3 seconds would probably have too much lag when used in a system in which the temperature fluctuates minimum to maximum to minimum in a period of 1 minute. According to frequency response theory for first order systems, the same thermometer would follow temperature fluctuations in a system with a period of 7 minutes, with an amplitude response of 0.1 percent.

"Repeatability" or reproducibility is mostly concerned with changes, temporary or permanent, which occur in the glass of the thermometer bulb (as it affects the volume) during manufacture and usage. For the better grades of thermometer glasses, these changes can be of order 0.02 to $0.2^{\circ} \mathrm{F}$, but may be much higher. For example, after 200 hours of heating at high temperature, changes as large as $21^{\circ} \mathrm{F}$ have been noted for thermometers made of glass unsuitable for the desired temperature range or improperly annealed [5]. Thus, ice or steam reference points are used periodically to check for such changes. Thermometers graduated aboue $300^{\circ} \mathrm{F}$ or precision thermometers with expected accuracy better than $0.2^{\circ} \mathrm{F}$ should have a refernce point. This is accomplished when the range includes an ice, steam, or other designated point, or the stem includes a short, auxiliary scale with a definite fixed point.

Maintenance and Calibration. Except for cleanliness, thermometers usually require no maintenance. For normal "field" usage, periodic checks of single point calibration at a designated reference temperature (ice or steam point, or other known reference temperature) are advisable. For use as a reference standard, calibration at NBS or another suitable laboratory is recommended. Before the thermometers are bought, a single point check, at leat, is advisable.

Failure Modes. Other than breakage, the most likely failures are due to mercury column separation and overheating. When overheated above the intended range, excess gas pressure can cause permanent distortion in the bulb configuration (volume) causing low readings. When mercury separation occurs, the bulb may be cooled in an ice-salt-water solution first, and if this is not successful; dry ice maybe used. See reference [5] for detailed procedures. Thermometers with evacuated stems are not recommended due to ease with which separation can occur. Some thermometers have interior bore effects causing erroneous readings after initial usage. 
Building EMCS Suitability. Liquid-in-Glass thermometers are quite suitable for EMCS programs for purposes of check and calibration purposes. The temperature ranges of building systems and those covered by thermometers are quite compatible. Consideration must be given, however, to the temperature range, best thermometer type, measurement accuracy desired, and whether the temperature to be measured is sufficiently steady. With regard to thermometer types, the partial immersion type appears best suited to well applications, with special attention paid to both well depth, well insulaticn, and thermometer immersion point. The well, of course, should be locted as near as possible to the appropriate on-line instrument when serving as a calibration check point. For checking room air or air side temperatures of HVAC equipment, the complete immersion type appears best suited. For offline calibrations using liquid baths as references, the total immersion type appears best suited.

\subsection{RESISTANCE THERMOMETERS (RTD)}

Operating Principles. The electrical resistance of pure metals increases with temperature, varying from about 0.3 to 0.6 percent change per degree $C$ (Celsius) at room temperature. Platinum is the most commonly used sensin element since it is available commercially in pure form and it is stable under different environmental conditions. A standard platinum thermometer serves as reference for the International Temperature Scale for the range $-190^{\circ} \mathrm{C}$ to $+600^{\circ} \mathrm{C}$. For temperatures above $0^{\circ} \mathrm{C}$, the resistance vs. temperature $(\mathrm{R} / \mathrm{T})$ relationship for platinum is given by (Callender-Van Dusen equation):

$$
R_{t} / R_{0}=1+\alpha\left[T-\delta\left(\frac{T}{100}\right)\left(\frac{T}{100}-1\right)\right]
$$

where $R_{T}$ is the resistance of $T^{\circ} \mathrm{C}, R_{0}$ is the resistance at $0^{\circ} \mathrm{C}$ and $\alpha$ and $\delta$ are constants determined by calibration for each individual element. Typical. values are $\alpha=0.000392^{\circ} \mathrm{C}^{-1}$ and $\delta=1.49^{\circ} \mathrm{C}$, and for small temperature ranges the $\mathrm{R} / \mathrm{T}$ relationship is very nearly linear. It is essential that the platinum be in an annealed and strain-free state. Thus, sensor design and construction are very important.

Nickel and copper sensors are also used. These are much cheaper than platinum. Nickel has an upper temperature limit of about $600^{\circ} \mathrm{F}$ and is appreciably nonlinear [6]. Its $\mathrm{R} / \mathrm{T}$ relationship is allegedly not as well know nor as reproducible as that of platinum [8]. Copper has a very linear $R / T$ relationship but has low resistance to oxidation above moderate temperatures and thus is limited to about $250^{\circ} \mathrm{F}$ [6]. The relatively low resistivity of copper also requires very sensitive voltage or current measurements.

Performance. Platinum, nickel and copper resistance thermometers are all well suited for building system applictions from the temperature range viewpoint, except for copper in applications above $250^{\circ} \mathrm{F}$, as mentioned above. Compared with thermocouples, resistance thermometers are capable of producing a significantly higher sensitivity. For example, the platinum element has about $0.4 \%$ resistance change $/{ }^{\circ} \mathrm{C}$. For a $100 \Omega$ sensor drawing a 
nominal current $2 \mathrm{~mA}$, the emf is $200 \mathrm{mV}$ and for a temperature change of $1^{\circ} \mathrm{C}$, the change in emf is $0.8 \mathrm{mV}$. Thus, the sensor is said to have a sensitivity of $0.8 \mathrm{mV} /{ }^{\circ} \mathrm{C}$ under these conditions. A copper-constantan thermocouple has a sensitivity of about $0.04 \mathrm{mV} /{ }^{\circ} \mathrm{C}$, or about $1 / 20$ th that of the resistance element. However, it is important that self heating in the resistance thermometer be negligible. This self heating effect varies with $\mathrm{I}^{2}$ and is governed by the rate of heat dissipated to the fluid. For measurement in still air, the self heating effect may necessitate such low values of I that the sensitivity of the resistance element is no longer as favorable.

The accuracy of the measured temperature will depend on many individual factors. Sources of errors may be classified as follows [9]:

- actual temperature of sensor different from temperature of the point of interest

- actual resistance of sensor different from measured resistance, and

- incorrect sensor resistance vs. temperature relationship being used (calibrated or uncalibrated).

Possible sources of temperature error include sensor self heating effects; improper sensor or well location where appreciable temperature gradients exist in the fluid being measured; insufficient immersion length of the sensor or well into th fluid (stem conduction effect); thermal inertia of sensor and well installation when fluid temperature varies with the time; and radiation between sensor or well and surroundings. Fluid (liquid) velocities in building systems are normally too low (say < 20fps) to induce significant frictional heating effects [10].

Possible sources of resistance error include lead wire and terminal contact resistance; variation of lead wire resistance with temperature; and errors in the measuring instrumentation system. Lead wire resistance errors are eliminated by use of elements with compensating leads ( 3 wire or 4 wire elements) and keeping all leads the same resistance when required by the bridge circuit. Special lead wire material is not required as with thermocouples.

When sensor elements are used interchangeably without individual element calibration, significant errors can be introduced depneding on sensor design and temperature level [11].

Thus, for builidng systems, typical inaccuracies may range from about $1.0^{\circ} \mathrm{F}$ up to 1 or $2^{\circ} \mathrm{F}$ or more depending upon the design and installation, and calibration procedures used.

The accuracy or uncertainty of the temperature measurement is often based on analysis of individual errors such as listed above and then results are combined into an overall uncertainty estimate. Another method is to compare results of measurements taken using two independent measurement 
systems when measuring temperature at the same point. In the former, the weakness is that individual errors may be quantified inaccurately or that important errors may be omitted. Also, the method of combining errors (linear vs. root-sum-square) is somtimes subject to judgment. In the latter, individual errors are not quantified, but combined effects of the individual errors (known and unknown) are taken into account through the measured systematic and random errors obtained from comparison of the performance of the two independent systems.

To illustrate the above, the following is a brief summary of calibration procedures used for computer-based differential temperature $(\Delta T)$ measurement systems used in a current energy monitoring system installed at NBS. The systems were designed to measure the $\Delta \mathrm{T}$ across hot water converter units, range 0 to $40^{\circ} \mathrm{F}$ ( 5 measuring units) and across chilled water coils, range 0 to $20^{\circ} \mathrm{F}$ ( 4 measuring units). Each measurement was to be accurate to an uncertainty of $+0.25^{\circ} \mathrm{F}$. Each system contained two resistance thermometer elements connected to a temperature transmitter unit having an output 4-20mA. The units were first calibrated in the temperature laboratory. Two of the nine systems failed to meet the $\pm 0.25^{\circ} \mathrm{F}$ uncertainty requirement. This is an example of the independent systems method where the laboratory reference temperature is the true value.

Next, each system was checked on-line in two parts as follows. In the first part, the resistance of the high and low temperature sensors were each measured under normal line flow conditions using a precision type digital multimeter and calibrated test leads. At the same time, the line temperatures were measured using suitable liquid-in-glass thermometers in nearby wells as the reference temperature. The reference temperatures were compared with the resistance sensor temperatures obtained using the manufacturer's $R / T$ data. This served as a check on the resistance sensors and included insta1lation, flow and any radiation effects on sensor performance. One sensor was found open-circuited, and with four sensors, the indicated temperature $(\mathrm{R} / \mathrm{T}$ data) differed from the reference temperature (1-iquid-in-glass thermometer) by more than $0.25^{\circ} \mathrm{F}$.

In the second part, the transmitter unit was checked by substituting a decade resistance box for each sensor. Thus, temperature inputs were simulated through decade resistance settings and use of the $\mathrm{R} / \mathrm{T}$ data. Transmitter output currents showed 3 transmitters in need of service or adjustment, and verified the results obtained from the previous laboratory calibrations. Checks of the complete system are made peiodically through comparison of on-line liquid-in-glass thermometer readings with the computed differential temperature output from the computer. This latter check is another example of using two independent systems to measure the same quantity.

Finally, no detailed analysis of individual errors was performed. It is believed, rather, that a most realistic estimate of uncertainty can only be obtained through demonstrations and checks of performance on-1ine such as these summarized above. 
The time constant for the sensor elements depends primarily on design and on heat dissipation rates. Time constants for transverse flow in water at $3 \mathrm{feet} / \mathrm{second}$ may vary from about 0.1 second to several seconds, and in low velocity flowing air up to a minute or more $[11,12]$.

Installation Precautions. The resistance element is a mechanically delicate instrument. Therefore, it should not be subject to excessive mechanical shock and vibration during shipment, installation and usage. Such may induce strains in the resistance wire itself which will normally increase the resistance [9]. For the nominal temperature ranges encountered in building systems, generally no damage would be induced from thermal shocks. The system design and installation should take into account the sensor immersion length, self heating effects, possible effects of the flow field temperature gradients, dynamic pressures, pressure/flow fluctuations, and radiation effects. Effects of temperature gradients and radiation are not likely to be significant for flow in insulated pipes. Shielding to minimize radiation effects may be necessary for elements installed in air side space in ACUs. Location of bare sensors far downstream of any positive displacement piston type pumps or quick acting control valves is preferred to avoid possible flow pulsation and vibration effects. Stem conduction error is minimized by sufficient sensor immersion [9], but for bare sensors the dynamic pressures due to impact forces from (high) transverse liquid flow velocity components may cause bending of the stem [8]. Sensors mounted in a well should have a close fit at the element proximity and, if possible, this space should be filled with a material of high thermal conductivity. The material in the well cavity should induce no endothermic or exothermic reactions which could possible influence sensor temperature. This may be a problem at high temperature, e.g. with certain oils at about $250^{\circ} \mathrm{F}$ or higher [9]. Self-heating effects can be minimized by calibrating and using the sensor at the same current values and in the same thermal environment (calibration on-line or the equivalent). Three-wire or four-wire configurations are used to eliminate effects of lead resistance.

Failure Modes. From experience gained in the NBS energy monitoring program, typical failures can include open resistance sensor elements, sensor element resistance "out-of-spec", and transmitter units out of adjustment. Sensor elements out-of-spec can sometimes be compensated through transmitter adjustment provided the element resistance is stable.

Maintenance and Calibration. For best accuracy, each sensor should be individually calibrated at reference temperature points which include or encompass the application range of interest. The constants in the Callender-Van Dusen equation can then be determined and the resistance at any temperature calculated. Otherwise, use of so called interchangeable sensors may degrade accuracy by 1 or $2^{\circ} \mathrm{F}$ or more, depending on sensor design and temperature range [11]. Possible calibration techniques have been reviewed previously under Performance. Periodic calibration should be performed, particularly to check sensor stability and transmitter (slope and intercept) performance. 
Building EMCS Suitability. Resistance thermometers are well suited for building system applications from the temperature range point of view and offer advantages over thermocouples because of significantly higher sensitivities $\left(\mathrm{mV} /{ }^{\circ} \mathrm{C}\right)$ and no reference junction equipment and special material lead wires required. With proper installation and handling, the stability of calibration is excellent for these applications, and because of the higher sensitivity possible compared to thermocouples, the measurement problem is less difficult. While resistance sensor elements are more costly than thermocouples, one manufacturer claims comparative costs may be about the same when considering special lead wires, higher gain amplifiers, and reference junction equipment needed for thermocouples [8]. Typical building system response rates are quite low relative to available probe response rates. Thus, probe response usually offers no problem.

\subsection{THERMOCOUPLES}

Operating Principles. As discovered by Seebeck in 1821, an electric current will flow in a closed circuit made of two wires of two dissimilar metals (thermocouple) when the wire junctions are at different temperatures. At the zero current or open circuit condition, a (thermal) electromotive force (emf) will exist at any point in the circuit. These effects are the principles on which temperature measurements by thermocouples are based. For single point measurements, the temperature of one junction (reference junction) must be known, usually the ice point or triple point of water, and the other junction (measuring junction) is exposed to the unknown temperature. For differential temperature measurements, each junction is exposed to a temperature and the $\Delta \mathrm{T}$ is measured directly. In this case, if the junctions between thermocouple extension wires and any copper connection wires are at the same temperature, no reference junctions are needed. To increase the sensitivity, thermocouple junctions are connected in series, called thermopiles. To observe the average temperature level at different locations, measuring junctions and reference junctions are connected in parallel. This requires the temperature/emf calibrations of all couples must be identical and linear, and all couples must have equal resistance.

Performance. See Performance Section of RTD for sensitivity comparisons of thermocouple and resistance bulb sensors. ANSI Standard MC 96.1 [14] lists the limits of errors for thermocouples, with reference junction at $0^{\circ} \mathrm{C}$. For type $\mathrm{T}$ thermocouples, in ordinary HVAC applications in the range, say $200^{\circ} \mathrm{F}$ maximum, the limit of error for standard wires (no special calibration) is $\pm 1.5^{\circ} \mathrm{F}$. If the combined error from the reference junction and temperature indicator is estimated at $+1^{\circ} \mathrm{F}$, a total error of $+2.5^{\circ} \mathrm{F}$ may be expected. When the measured medium is $400^{\circ} \mathrm{F}$, the expected error may be larger than $+4^{\circ} \mathrm{F}$. The response time is very short. The time constant for grounded sheathed thermocouples is of the order of seconds in air measurement of HVAC applications.

Calibration and Maintenance. Calibration of thermocouples after some period of operation should be performed on-line using a calibrated liquid-in-glass thermometer, RTD, or another thermocouple as the reference. The effects of 
wire connections, aging of the wire and temperature gradients along the wires are all accounted for by this calibration method. More than one point in the operating temperature range should be calibrated. Calibration wells should be installed in pipes near the sensing wells. From the standpoint that the temperature of the medium is seldom stable, a calibrated thermocouple serving as the reference may be preferred because of its similar thermal mass with the thermocouple to be calibrated, although the calibration uncertainty using thermocouples is higher than those of the liquid-in-glass thermometer or RTD. The reference thermocouple should be calibrated in the laboratory often. No other maintenance is required.

Installation Precautions. When a thermocouple is used for measuring the temperature of water and steam, a thermowell should be installed to protect the thermocouple and to provide pipe integrity. The immersion depth of the thermowell should be long enough to minimize the conduction loss of the well and thermocouple wires. An often used practice is to give a minimum depth of ten times the outside diameter of the well. The thermowell extension should be provided for pipe insulation. For small diameter pipes, the thermowell should be installed at an angle or in a tee to accomodate the required well depth. Good contact between the thermocouple and thermowe 11 is needed to improve the response to temperature change. For air temperature sensing, a sheathed thermocouple with a sealed end should be used to provide the necessary support and protection from mechanical, chemical and moisture damage. Excessive temperature difference between the two terminals of thermocouples and extension wires should be avoided. Shielding and grounding of the thermocouple extension wires to reduce interference from other electrical and magnetic fields are important. These wires should not be installed in the same conduit with other electrical wires and they should not run in parallel to or close to building power wires. In locating the sensors in pipes, ducts and air handling units, precautions to reduce radiation effects should be observed as described above for resistance thermometers.

Failure Modes. Failure modes of thermocouples and the possible causes of failure are listed in detail in Table 33 of [13].

Building EMCS Suitability. The thermocouple has a relatively simple constuction and is quite flexible for field installation. The small mass gives a very short response time. The type $T$ thermocouple may be used for building HVAC applications because of its suitable operating temperature range and high resistance to atmospheric moisture. Thermocouples have no self heating problems, unlike some other temperature measuring sensors, such as resistance bulbs. Thermocouples are particularly well suited for measurement of temperature differentials, such as those in chilled and hot water supply and return lines, because $\Delta \mathrm{T}$ is measured directly without the use of reference junction equipment or temperature ice bath and copper extension wires are used from the point of temperature sensing to the point of emf measurement. 


\subsection{SALT-PHASE TRANSITION TYPE HYGROMETER}

Operating Principles. A thin-walled metal tube is wrapped with a cloth sleeve impregnated with a hygroscopic salt such as lithium chloride. A bifilar winding of two electrodes is wound outside the cloth sleeve and is connected to low voltage, alternating current. Since the electrical resistance of this element varies with the concentration of the salt solution, an equilibrium temperature is reached when the rate of moisture absorption of the salt equals the moisture evaporation due to the heat generated in the salt by the electric current. This temperature is related to the partial pressure of the water vapor content and the dew point of the air and is measured by a temperature sensor inside the metal tube to give the dew point of the air. Theoretically this hygrometer does not need calibration, if the salt is not contaminated.

Performance. The operating range of this hygrometer is between $0^{\circ}$ and $160^{\circ} \mathrm{F}$ dew point and is limited to relative humidity above 15\%. The typical error is $+3^{\circ} \mathrm{F}$ dew point above $10^{\circ} \mathrm{F}$ dry bulb; it may go as high as $6^{\circ} \mathrm{F}$ below $10^{\circ} \mathrm{F}$ dry bulb. The response time depends on the dew point of the air. For dew points above $10^{\circ} \mathrm{F}$, the response time (90\%) is below 3 minutes.

Calibration and Maintenance. Like any other measuring equipment, the buyer should require the contractor to demonstrate that the hygrometer is calibrated prior to acceptance. Most hygrometers need to be calibrated periodically after they are installed. The interval of calibration of this type depends on the quality and the stability of the thermometer, and the quality of the air the sensor is required to measure. The calibration includes calibrating the temperature sensor and checking the salt element temperature against the temperature-dew point curve. When its salt element is exposed to humid air, the hygrometer should always be energized (element heated) so that the element would not absorb excess amounts of moisture causing the salt solution to run off. Retreatment of the salt element is necessary if sensor current is interrupted for a prolonged period of time, since the salt element may absorb excess moisture. or become contaminated. The retreatment intervals depends on the air quality the element senses. One reference, [15], suggests 90 to 100 days and one manufacturer recommends at least twice a year [16]. The hygrometers used in NBS environmental chambers have been retreated every one to two years. Retreatment usually is done by washing off the contaminated salt, applying fresh salt solution and drying.

Installation Precautions. Excessive air velocity, say above 50 feet per minute, at the salt element may reduce the temperature at the thermometer and give lower dew point readings [17]. Some manufacturers recommend using shields. When installed outdoors, a weather hood should be provided to protect the sensor from contacting rain water. Condensation on the salt element may change the calibration. In selecting sensor locations, avoid locations where the following contaminates are present in the air: hydrogen sulfide, sulphur dioxide, acid vapor, chlorine, ammonia, alkaline vapors, acetylene, ethylene oxide, salt contaminated air, alcohols and glycol vapors. 
Failure Modes. Total failure of this hygrometer is not frequent but drifting of readings due to salt element contamination is common.

Building EMCS Suitability. Since the dew point is actually measured by dry bulb temperature, this type of hygrometer is easily used in EMCS systems to sense the outdoor dew point. The lower range of $15 \% \mathrm{RH}$ of the instrument should not be a limiting factor in building HVAC applications. The response time is slower than some other hygrometers. It may not be suitable for space dew point monitoring where the humidifier response is fast, such as for the dry steam type humidifier. Frequent maintenance is required.

\subsection{IMPEDENCE TYPE HYGROMETER}

Operating Principles. There are various arrangements and materials in construction of this kind of hygrometer. Some are based on the principle that the electrical resistances and/or capacitances of certain hygroscopic materials change with the concentrations of the material. Others are based on the fact that the electrical resistances and/or capacitances of the surfaces of certain materials vary with the moisture they adsorb. Since the moisture absorption and adsorption are functions of the relative humidity of the space, the electrical impedance provides a measure of the space relative humidity. The compensation for the effect of temperature must be made for some transducers, because temperature variations may also change the electrical impedance. However, some sensors are practically independent of temperature in the temperature range of HVAC application. Multiple sensors covering different ranges and wide range single sensor are available. Alternating current is often used in the measurement to avoid polarization of the sensors.

Performance. The performances vary widely among the sensors of this type due to their diversified arrangements and materials used. Individual hygrometer performances should be examined for their application. Sensors covering the general range of $10 \%$ to $99 \% \mathrm{RH}$ and in the $\mathrm{HVAC}$ application range of dry bulb temperature are numerous. The response time ( $63 \%$ of change) is much shorter than the salt-phase transition type hygrometer, ranging from a few seconds to less than a minute with adequate ventilation rate at the sensor. Dunmore type (electrolytic) hygrometers may be used in the range of $2 \%$ to $99 \% \mathrm{RH}$ and have accuracies of $+3 \% \mathrm{RH}$. Aluminum oxide (surface resistance and capacitance) hygrometers mày be used between $5 \%$ and $100 \%$ RH with accuracies of $\pm 3 \% \mathrm{RH}$ and they have very 1 ittle temperature effect. Dielectric film (capacitance) hygrometers are usually in the range of $20 \%$ to $80 \% \mathrm{RH}$. The accuracy may be no better than $\pm 5 \% \mathrm{RH}$. The errors in impedence type hygrometers are due mainly to hysterisis.

Calibration and Maintenance. Performance of these types of hygrometers are determined empirically, and they must be calibrated individually to insure their accuracy. As with the salt-phase transition type hygrometers, the recalibration period depends on the air quality and sensor handling techniques. A proper calibration schedule should be established by observing the drift characteristics with short calibration intervals at the beginning. Calibration in laboratories can be done by: (a) generating humidity at known levels, such as two-pressure systems; or (b) using saturated salt 
solution chambers where the humidity level is known for a certain temperature. Calibration services are available from the National Bureau of Standards and other standards laboratories. Field calibration, which may be more suitable for ElCS systems, may be performed by using a newly laboratory calibrated hygrometer as a reference standard. Some hygrometers have temperature sensors for correcting temperature effects. They should be calibrated according to their requirements. Other than calibration and maintenance of any filter equipment, these sensors require little maintenance. The manufacturer's maintenance manual should be consulted.

Installation Precautions. Humidity sensors should be handled carefully so that they will not be damaged or contaminated. Chemical contamination by acids, sulfur compounds, or certain solvents may damage the sensors or shift their calibrations. In locating hygrometers for outdoor air humidity sensing, locations near cooling towers, boiler stacks or other chemical exhausts should be avoided. Condensation or water may shift the calibration of some sensors of this type. Rain protection should be provided.

Failure Modes. Similar to salt-phase transition type hygrometer.

Building EMCS Suitability. Depending on the humidity range, some sensors are not suitable for outdoor use, such as the dielectric film capacitance type. The wide range Dunmore type can be used in either outdoor, duct, or space sensing. The fast response of these hygrometers may be used for any type of building humidification control.

\subsection{DIMENSIONAL CHANGE HYGROMETER}

Operating Principles. Many hygroscopic materials change length or volume when responding to the relative humidity level of the air. Examples are hair, wood, paper, and cellulose. In one group of hygrometers, using carbon particle coatings or impregnating such materials, a humidity induced change in dimension causes a change in the electrical resistance of the sensors. Since the electric conductivity is due to electronic rather than electrolytic effects, there is little polarization of the sensor and either AC or DC excitation may be used. This discussion concerns this type of carbon sensor.

Performance. The sensitivity is low at low relative humidity. It increases gradually after approximately $40 \% \mathrm{RH}$. Between $90 \%$ and $100 \% \mathrm{RH}$, the humidityresistance curve flattens or dips. Therefore, the useful range is somewhere between $20 \%$ and $90 \% \mathrm{RH}$. Hystersis is about $\pm 3 \% \mathrm{RH}$ average with higher values in the lower humidity range. Typical accuracy is no better than $\pm 5 \%$ RH. The response time is typically less than 1 minute ( $63 \%$ of change).

Calibration and Maintenance. Calibration should be performed frequently, perhaps every three to six months. Since the resistance of the carbon sensor is also temperature dependent to some extent, the hygrometer should be calibrated for the existing site temperature range. No other maintenance is required. 
Installation Precautions. General installation precautions for all humidity sensors should be observed, i.e., avoiding locations where temperature variation is large and air quality is poor.

Failure Modes. Similar to other types of hygrometers described in Sections 4.1 and 4.2 .

Building EMCS Suitability. This type of hygrometer is suitable for space and return duct installations because of its fast response and relatively maintenance free (except for calibration) characteristics. The temperature dependency and the humidity-resistance curve dipping at high humidity preclude its use for outdoor measurements. 


\section{REFERENCES}

1. Fluid Meters, Their Theory and Application; 6th edition, ASME, 1971.

2. L.K Spink, Principles and Practice of Flow Meter Engineering, 9 th edition, The Foxboro Company, 1967.

3. ISA, Installation \& Calibration of Turbine Flow Meters, Recommended Practice, RP 31.1, 1972.

4. M. R. Shafer, Performance Characteristics of Turbine Flow Meters, ASME Journal of Basic Engineering, December 1962.

5. Liquid-in-Glass Thermometry, NBS Monograph 150, January 1976.

6. Fundamentals of Temperature, Pressure and Flow Measurements, Robert P. Benedict, 2nd Edition, John Wiley and Sons 1977.

7. Temperature Measurements, Standard Measurements Guide, ASHRAE Standard 41.1-74, 1974.

8. Rosemount Engineering Company (REC), Platinum Resistance Temperature Sensors, Bulletin 9612, Rev. B, 1962.

9. John L. Riddle, George T. Furukawa, and Harmon H. Plumb, Platinum Resistance Thermometry, NBS Monograph 126, Apri1 1973.

10. REC, Frictional Heating of the Temperature Sensor for the Case of Moving Fluids, Bulletin 8628, F.D. Werner \& R.V. DeLeo, undated.

11. REC, Industrial Temperature Sensors, Platinum Resistance Type, Bulletin 1042, Rev. A, 1967.

12. Minco Products, Inc., Minco Temperature Sensors, Bulletin TS-100, undated.

13. Manual on the Use of Thermocouples in Temperature Measurement, Special Technical Publication 470A, American Society for Testing and Materials, 1974.

14. American National Standard for Temperature Measurement Thermocouples. ANSI-MC96.1, Instrument Society of America, 1975.

15. Kurt S. Lion, Instrumentation in Scientific Research, McGraw-llill Book Company, 1959.

16. Honeywe11 SSP129A-D DewProbe Sensor Instructions, REv. 1969.

17. Arnold Wexler, Electric Hygrometers, NBS Circular 586, 1957. 


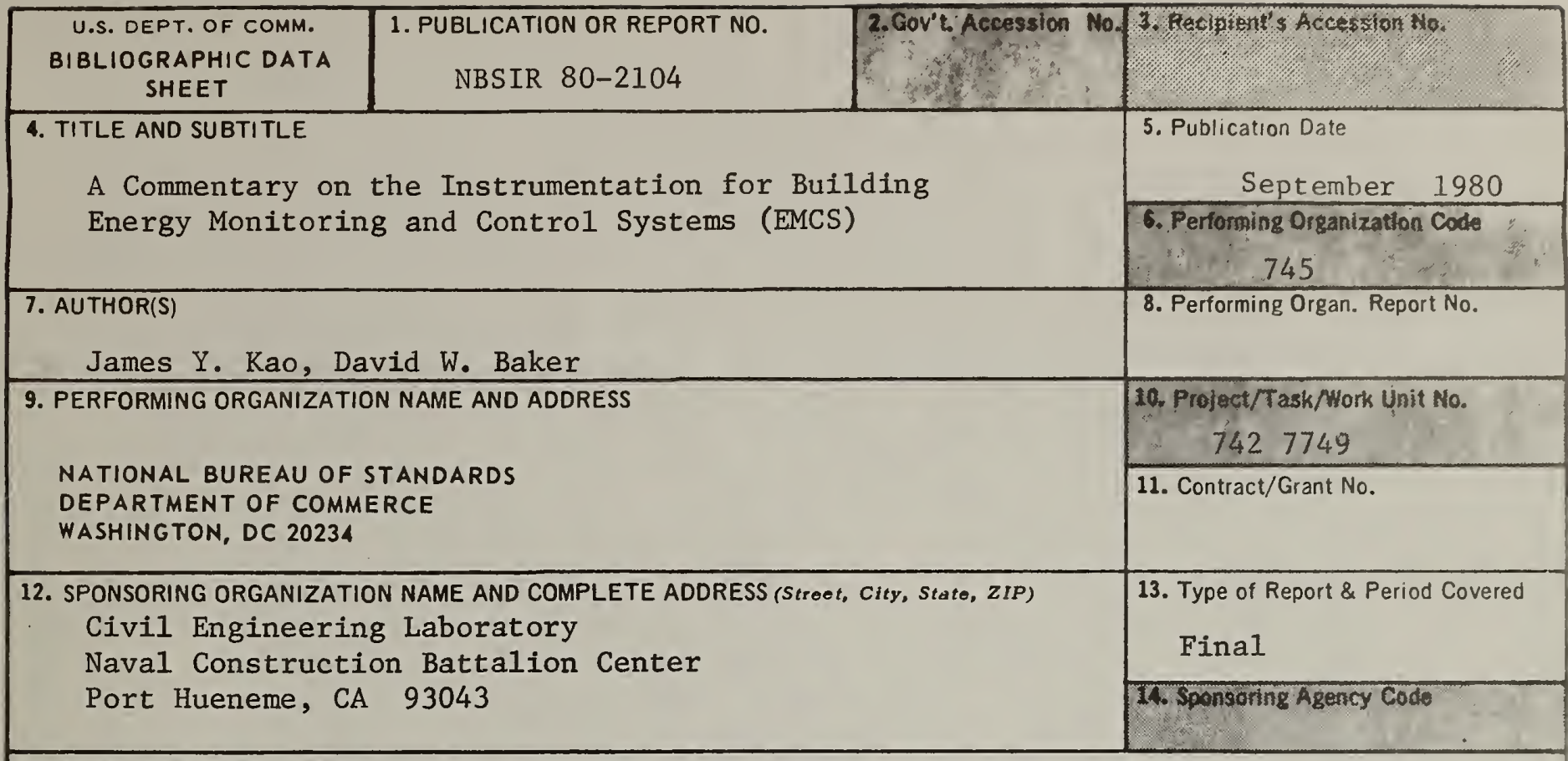

15. SUPPLEMENTARY NOTES

$\square$ Document describes a computer program; SF-185, FIPS Software Summary, is attached.

16. ABSTRACT (A 200-word or loss factual summary of most significant information. If document includes a significant bibliography or literature survey, mention it hero.)

This paper reviews sensors generally used for building energy monitoring and control systems (EMCS). The sensor operating principles, performances, calibration, maintenance, installation precautions, failure modes and their suitability for building EMCS use are discussed. Sensors covered in the paper include orifices, flow nozzles, Venturis, vortex shedding meters, and turbine meters for flow measurements, liquid-in-glass thermometers, resistance thermometers, and thermocouples for temperature measurements, and salt-phase transition hygrometers, impedance hygrometers, and dimensional change hygrometers for humidity measurements.

17. KEY WORDS (six to twelve entries; alphabotical order; capitalize only the first letter of the first key word unless a proper name; separated by semicolons)

Building energy monitoring; instrumentation; sensors

18. AVAILABILITY

Ex Unlimited

For Official Distribution. Do Pot Release to NTIS

Order From Sup. of Doc., U.S. Government Printing Office, Washington, DC 20402, SD Stock No. SNÓ03-003-

$[\bar{X}]$ Order From National Technical Information Service (NTIS), Springfield, VA. 22161

\begin{tabular}{|l|c|}
\hline $\begin{array}{l}\text { 19. SECURITY CLASS } \\
\text { (THIS REPORT) } \\
\text { UNCLASSIFIED }\end{array}$ & $\begin{array}{c}\text { 21. NO. OF } \\
\text { PRINTED PAGES }\end{array}$ \\
\hline $\begin{array}{l}\text { 20. SECURITY CLASS } \\
\text { (THIS PAGE) }\end{array}$ & 27 \\
UNCLASSIFIED & $\$ 6.00$ \\
\hline
\end{tabular}




